\title{
FOXP3 Isoforms and Human Regulatory T Cells
}

\author{
Rebeca Santos ${ }^{1}$ and Baohua Zhou ${ }^{2}$ \\ ${ }^{1}$ Indiana University School of Medicine; ${ }^{2}$ Indiana University School of Medicine, \\ Department of Pediatrics
}

\begin{abstract}
Background:
Regulatory T-cells (Tregs) are critical to maintaining immune tolerance, thus prevent autoimmunity and allergic diseases. The master transcription factor FOXP3, expressed in humans as two isoforms through mRNA alternative splicing, controls the development and function of Tregs. However, the functions of the two isoforms remain unclear. We hypothesized that the oligonucleotides, developed by the lab, would efficiently shift FOXP3 to its shorter isoform, lacking exon 2 (FOXP3 $\triangle \mathrm{E} 2$ ), which enables us to study how the FOXP3 $\triangle \mathrm{E} 2$ isoform affects Treg function.
\end{abstract}

\section{Methods:}

Human peripheral blood mononuclear cells (PBMC) were obtained from healthy donors and cultured in the presence of $1 \mu \mathrm{M}$ isoform-shifting oligonucleotides for three weeks. Expression of the FOXP3 isoforms, CD25, CTLA-4, CD40L, was examined through flow cytometry. Another goal was to study how the FOXP3 $\triangle \mathrm{E} 2$ isoform shift affects the Treg response to inflammatory cytokines.

\section{Results:}

The oligonucleotides are highly effective in shifting the FOXP3 to the FOXP3 $\triangle E 2$ isoform. The majority of Tregs express only the FOXP3 $\triangle \mathrm{E} 2$ isoform after two weeks of culturing in $1 \mu \mathrm{M}$ oligo and had reduced expression of CD25, which plays a role in Treg differentiation, function, and homeostasis. Tregs display reduced expression of CTLA-4, a negative regulator of T-cell activation, but elevated CD40L, which costimulates and regulates the immune response. The FOXP3 $\triangle E 2$ isoform shift also sensitizes the Tregs to proinflammatory cytokine stimulation, allowing for increased IL-4, IL-17A, and IFN-Y production.

\section{Conclusion:}

Our studies demonstrate that FOXP3 $\triangle \mathrm{E} 2$ Tregs are less stable because of the lower CD25 expression and less suppressive as determined by lower CTLA-4 but higher CD40L expression. In the inflammatory environment, the FOXP3 $\triangle \mathrm{E} 2$ Tregs also produce more inflammatory cytokines, which further reduces their regulatory function. This study establishes an understanding of FOXP3 $\triangle E 2$ Tregs' role in autoimmunity and provides a potential novel target to treating autoimmune diseases.

\section{Acknowledgements:}

This study was funded, in part, with support from the Immunology and Infectious Diseases Training Program Grant funded, in part by T32 Al 060519 from the National Institutes of Health to RS and NIH Al159804 to BZ. 